\title{
Differentiation of ductal carcinoma in situ versus fibrocystic changes by magnetic resonance imaging: are there pathognomonic imaging features?
}

Acta Radiologica

2017, Vol. 58(10) 1206-1214

(C) The Foundation Acta Radiologica 2017

Reprints and permissions:

sagepub.co.uk/journalsPermissions.nav DOI: $10.1177 / 0284185117690420$ journals.sagepub.com/home/acr (SAGE

\author{
Matthias Dietzel',*, Clemens G Kaiser ${ }^{2, *}$, Evelyn Wenkel', \\ Paola Clauser ${ }^{3}$, Michael Uder', Rüdiger Schulz-Wendtland' and \\ Pascal AT Baltzer ${ }^{3}$
}

\begin{abstract}
Background: In breast magnetic resonance imaging (MRI), the diagnosis of ductal carcinoma in situ (DCIS) remains controversial; the most challenging cause of false-positive DCIS diagnosis is fibrocystic changes (FC).

Purpose: To search for typical and pathognomonic patterns of DCIS and FC using a standard clinical MRI protocol. Material and Methods: Consecutive patients scheduled for breast MRI (standardized protocols@ I.5T: dynamicTI-GRE before/after Gd-DTPA [0.I mmol/kg body weight (BW)]; TI-TSE), with subsequent pathological sampling, were investigated. Sixteen MRI descriptors were prospectively assessed by two experienced radiologists in consensus (blinded to pathology) and explored in patients with DCIS $(n=77)$ or FC $(n=219)$. Univariate and multivariate statistics were performed to identify the accuracy of descriptors (alone, combined). Furthermore, pathognomonic descriptor-combinations with an accuracy of $100 \%$ were explored ( $\chi^{2}$ statistics; decision trees).

Results: Six breast MRI descriptors significantly differentiated DCIS from FC ( $P_{\text {corrected }}<0.05$; odds ratio $\left.<7.9\right)$. Pathognomonic imaging features were present in $33.8 \%(n=100)$ of all cases allowing the identification of $42.9 \%$ of FC $(n=94)$.

Conclusion: Pathognomonic patterns of DCIS and FC were frequently observed in a standard clinical MRI protocol. Such imaging patterns could decrease the false-positive rate of breast MRI and hence might help to decrease the number of unnecessary biopsies in this clinically challenging subgroup.
\end{abstract}

\section{Keywords}

Magnetic resonance imaging, ductal carcinoma in situ (DCIS), fibrocystic changes, diagnostic accuracy, breast cancer

Date received: 17 March 2016; accepted: 15 December 2016

\section{Introduction}

Breast cancer can be diagnosed at the invasive or the pre-invasive stage. The latter is known as ductal carcinoma in situ (DCIS) and has a much more favorable prognosis with a 20-year breast cancer-specific mortality of $3.3 \%$ (1). DCIS is the fourth leading cancer among women in the United States and an accurate diagnosis is important (2).

Next to mammography and ultrasound, magnetic resonance mammography (MRM) is the most important imaging method of the female breast (3). It provides both excellent soft tissue contrast and, due to dynamic
'Department of Radiology, University Hospital Erlangen-Nürnberg, Erlangen, Germany

${ }^{2}$ Institute of Clinical Radiology and Nuclear Medicine, University Medical Center Mannheim, Mannheim, Germany

${ }^{3}$ Department of Biomedical Imaging and Image-Guided Therapy, Division of Molecular and Gender Imaging, Medical University of Vienna, Vienna, Austria

*Equal contributors.

\section{Corresponding author:}

Pascal Baltzer, Department of Biomedical Imaging and Image-Guided Therapy, Division of Molecular and Gender Imaging, Medical University of Vienna, Waehringer-Guertel 18-20, Vienna General Hospital (AKH), Vienna, Austria.

Email: pascal.baltzer@meduniwien.ac.at 
contrast-enhanced techniques, functional information about tissue vascularity. This enables imaging of breast cancer with the highest sensitivity and good specificity using standard clinical protocols $(3,4)$.

Compared to invasive ductal cancers, DCIS shows a different growth pattern within the ducts and typical neovascularization: Hereby tumor cells can directly release angiogenic factors resulting in a rim of microvessels adjacent to the basement membrane of affected ducts or indirectly via recruitment of accessory cells leading to diffuse stromal vascularity. Additionally, slow enhancement of the actual DCIS ducts can also be due to gadolinium entering the leaky ducts (5-7). Taking this into account, it is not surprising that MRM features of invasive breast cancers and DCIS can show obvious differences.

Space-occupying benign lesions, such as fibroadenomas, do not typically account for a significant proportion of false-positive DCIS diagnoses. It is more the presence of fibrocystic breast changes (FC) that raises the rate of false-positive MRM diagnoses in DCIS patients and makes it remarkably difficult. FC is a benign histopathological condition characterized by breast lumps and cystic components of various size. Clinically, tenderness and pain might be present. On MR imaging (MRI), the presence of FC can lead to poorly delineated and significantly enhancing lesions which mimics DCIS $(8,9)$.

To date, there are still insufficient systematic data on the typical appearance of DCIS and its differentiation from FC $(8,9)$. Accordingly, this study was designed to identify typical and pathognomonic patterns of DCIS and FC using a standard clinical MRM protocol.

\section{Material and Methods}

\section{Patients}

Informed consent for this cross-sectional study was waived by the authors' institutional ethical committee. Data were prospectively collected at the breast center of the Hospital of the Friedrich Schiller University, an academic tertiary care institution over 12 years. This breast center consists of the Departments of Gynecology, Oncology, Radiology, Pathology, and Radiation Oncology. Diagnostic workup, treatment, and followup of patients with suspected or proven breast cancer are done in close accordance with national and international guidelines $(3,4,10)$. Therapeutic decisions are established in multidisciplinary consensus.

Indication of MRM were unclear findings upon conventional imaging, including such cases as presence of suspicious lymph nodes and missing mammography/ ultrasound correlate in the breast as well as presence of unilateral bloody discharge without conventional imaging findings (BI-RADS 0-3), or suspicious conventional findings (BI-RADS 4 and 5). Exclusion criteria were condition after biopsy, surgery, chemotherapy, or radiation therapy up to one year prior to MRM and BI-RADS 6 to eliminate biological bias. The latter is particularly relevant in the MRM assessment of subtle lesions such as DCIS.

All patients receiving histopathological verification of DCIS (without microinvasive component) or FC after breast MRM were selected from the institutional database and formed the dataset of the present study. Histopathology workup was performed by boardcertified breast pathologists using specimens from image-guided biopsy (core biopsy, vacuum-assisted biopsy) or surgical excision. 14-Gauge core biopsy under sonographical guidance was performed if lesions could be visualized by ultrasound. Otherwise, 12-Gauge VABB was used either stereotactically or MRI-guided; MRI guidance was exclusively used in cases of MRM-only lesions. Surgery was performed in case of discrepant findings between imaging and histology, in lesions with uncertain malignant potential upon pathological examination, in rare cases according to patients' preference, and in all malignant lesions.

\section{MR protocol}

Clinical MR scanners at $1.5 \mathrm{~T}$ were used with dedicated bilateral breast coils as provided by the vendor (Magnetom Avanto and Sonata; Siemens Healthcare, Erlangen, Germany). Patient position was prone and the standard scan orientation was axial. We applied a standardized clinical imaging protocol according to current technical recommendations $(4,11)$.

A T2-weighted (T2W) turbo spin echo sequence was acquired to assess morphological tissue details. Technical parameters were: TR, $4000-8900 \mathrm{~ms}$; TE, 200-300 ms; flip angle (FA), 90 ; slice thickness, 3$4 \mathrm{~mm}$; and matrix, $256 \times 512$ pixels.

Dynamic contrast-enhanced imaging was done both to provide morphological tissue details and to image its vasculature: Spoiled dynamic T1-weighted (T1W) gradient echo sequences with a temporal resolution of $60 \mathrm{~s}$ were used. After the pre-contrast scan, contrast agent was injected intravenously. The injection protocol was standardized with regard to the contrast media (Gd-DTPA, Magnevist; Bayer HealthCare, Berlin, Germany), flow $(3 \mathrm{~mL} / \mathrm{s})$, and the subsequent final saline flush $(30 \mathrm{~mL})$. After a delay of $30 \mathrm{~s}$, the seven post-contrast scans were acquired. Post-processing included generation of subtracted images without the use of motion correction. Technical parameters were: TR, 100-110 ms; TE, $5 \mathrm{~ms}$; FA, $80^{\circ}$; slice thickness, 3-4 mm; field of view, $350 \mathrm{~mm}$; and matrix, $256 \times 384$ pixels. 


\section{Reading}

Readers were blinded to histopathological results and experienced in breast MRM (>5000 examinations, 10 years). Patient history and imaging reports (i.e. mammography and/or ultrasound) were available to the readers.

Readers strictly followed the prospectively defined reading protocol. This approach was developed and has been validated in numerous institutions regarding inter-observer variability (12), diagnostic accuracy (13-15), and clinical subgroups (16-18). In every patient, the same hanging protocol was used, with the same windows settings (levels of center, width, magnification, etc.).

A checklist of 16 MRM descriptors was assessed retrospectively to describe MRM patterns of each lesion within the dataset. A lesion was hereby defined as an enhancing area on the first or second post-contrast scan. Note that this reading approach does not require a dichotomization of lesions into mass or non-mass lesions (11). All MRM descriptors were rated qualitatively by both radiologists in consensus. Initially, lesion size was evaluated by measuring the largest diameter on contrastenhanced T1 scans (first or second post-contrast scan).

The checklist included dynamic parameters (wash-in; wash-out, plateau, continued increase; blooming sign). The latter was positive if a lesion showed smooth margins in the first post-contrast scan that became increasingly blurry during the delayed phase. Morphologic descriptors included shape, signal intensity (T2 and T1 before contrast agent compared to the surrounding breast parenchyma). Vessels leading directly to the tumor were classified as "adjacent vessel sign." The hook sign was rated positive if a dendrite could be delineated arising from the lesion's center with direct connection to the pectoral muscle. More details on the descriptors is given in the supplementary materials and can also be found in previous issues of Acta Radiologica (17).

\section{Data analysis}

The presence of each MRM descriptor and the reference standard was documented in the database on a categorical scale level. In a first step, all MRM descriptors were correlated with the reference standard using uni- and multidimensional crosstabs. The diagnostic accuracy of single descriptors was identified by means of sensitivity, specificity, and likelihood ratios (positive/ negative: $\mathrm{LR}+(\mathrm{LR}-$ ), as well as the diagnostic odds ratio (DOR). Chi-square statistics were performed to identify significant differences in each MR descriptor stratified by the reference standard.

In a second step, decision trees (DT) were generated. DT generate simple, intuitive-to-follow decision rules and results can be directly applied to clinical practice.
For this purpose, the DT algorithm splits the whole dataset into "nodes." A node is hereby defined by a certain descriptor combination. This characteristic descriptor combination can be assigned a certain likelihood for DCIS and FC. Accordingly, a node represents a diagnostic criterion, just like a single MRM feature (e.g. hook sign).

Furthermore, DT enable the identification of subgroups with maximum diagnostic certainty $\left(D C_{\max }\right)$. This allows to identify pathognomonic descriptor combinations, enabling $100 \%$ accurate classification of lesions either as "DCIS" or "FC" as intended by the research question.

The CRT (classification and regression tree) growth method was applied for this purpose. All MR descriptors were set as input variables, whereas histology served as a classification variable. Ten-fold crossvalidation was performed to confirm the results on an independent dataset and to accurately assess the predictive power of the CRT model on unknown cases.

For all statistical tests, the null hypothesis $\left(\mathrm{H}_{0}\right)$ was defined as "no difference between DCIS and FC" in the given MR descriptor. Hypotheses were tested in a two-sided manner at $\alpha=5 \%$. To control for $\alpha$-accumulation, Bonferroni correction was applied.

\section{Results}

\section{Patients and reference standard}

A total of 296 patients were included (mean age, 52 years; standard deviation [SD], 11.8 years). Of these, 77 were diagnosed with DCIS (mean age, 56.5 years; SD, 11.2 years) and 219 patients were diagnosed with FC (mean age, 50.4 years; SD, 11.6 years). Lesion size showed no differences between the subgroups of DCIS and FC lesions, excluding a confounding effect on final MR diagnosis $(P=0.2$; see also supplementary material).

\section{Assessment of individual MRM descriptors}

In total, $37.5 \%$ of all descriptors (6/16) were able to significantly differentiate DCIS from FC. These were wash-in $\left(P_{\text {corr }}=0.0006\right)$, blooming sign $\left(P_{\text {corr }}<\right.$ $0.0001)$, wash-out $($ Pcorr $<0.0001)$, adjacent vessel sign $\left(P_{\text {corr }}=0.001\right)$, signal intensity T1 $\left(P_{\text {corr }}=0.02\right)$, and shape $\left(P_{\text {corr }}=0.002\right)$.

Single descriptors reached diagnostic odds ratios of up to 7.9 (intermediate to strong wash-in), 4.2 (blooming sign), 3.9 (wash-out) and 3.8 (iso- or hypointense signal on T1). The highest positive likelihood ratios $(\mathrm{LR}+)$ for predicting the presence of DCIS were observed for blooming sign ( $\mathrm{LR}+2.8)$, adjacent vessel sign $(L R+2.6)$, and wash-out $(L R+2.5)$. The 
highest specificity was observed for adjacent vessel sign $(88.6 \%)$, blooming sign $(85.4 \%)$, and wash-out $(80.8 \%)$. Further details are given in Table 1 and the supplementary material.

\section{Multivariate analysis}

Significant descriptor-combinations were identified to differentiate DCIS from FC. For instance, if a lesion showed wash-out plus irregular shape plus iso- or hypointense signal intensity on $\mathrm{T} 1$ (node 17; $P<0.0001$ ), the lesion was likely to be malignant $(\mathrm{LR}+7.5)$, with high overall accuracy (DOR, 9.9). Accuracy and definition of nodes that reached an $\mathrm{OR} \geq 5$ are summarized in Table 1 .

A total of seven nodes fulfilled $\mathrm{DC}_{\max }$ and are summarized in Table 2. Based on such nodes, $33.8 \%$ of all lesions $(n=100)$ were classified with a certainty of $100 \%$ and with a zero error rate. If stratified by histology, $42.9 \%$ of FC $(n=94)$ and $7.8 \%$ of DCIS $(n=6)$ could be diagnosed with $\mathrm{DC}_{\max }$. Examples of $\mathrm{FC}$ and DCIS are shown in Figs. 1-4.

\section{Discussion}

Since the landmark paper by Kuhl et al. in 2007, the high sensitivity of MRM for the detection of DCIS has been established (19). Nevertheless, the clinical role of

Table I. MR patterns for the differential diagnosis of DCIS from FC.

\begin{tabular}{|c|c|c|c|c|c|}
\hline Descriptor & Sensitivity & Specificity & $\mathrm{LR}+$ & LR- & DOR \\
\hline Wash-in & 96.1 & 24.2 & 1.3 & 0.2 & 7.9 \\
\hline Blooming sign & 41.6 & 85.4 & 2.8 & 0.7 & 4.2 \\
\hline Wash-out & 48.1 & 80.8 & 2.5 & 0.6 & 3.9 \\
\hline $\mathrm{SI} \mathrm{T} 2$ & 94.8 & 17.4 & I.I & 0.3 & 3.8 \\
\hline Adjacent vessel sign & 29.9 & 88.6 & 2.6 & 0.8 & 3.3 \\
\hline Shape (irregular) & 68.8 & 57.5 & 1.6 & 0.5 & 3 \\
\hline $\begin{array}{l}\text { Node 17: wash-out }+ \\
\text { irregular } \\
\text { shape }+ \text { SI TI }\end{array}$ & 27.3 & 96.3 & 7.5 & 0.8 & 9.9 \\
\hline $\begin{array}{l}\text { Node } 17+\text { absent } \\
\text { Hook sign }\end{array}$ & 19.5 & 96.3 & 5.3 & 0.8 & 6.4 \\
\hline $\begin{array}{l}\text { Node 5: wash-out or } \\
\text { plateau + irregular } \\
\text { shape }\end{array}$ & 45.5 & 85.4 & 3.1 & 0.6 & 5 \\
\hline Node $5+$ SI TI & 45.5 & 87.7 & 3.7 & 0.6 & 5.9 \\
\hline
\end{tabular}

Given are six single descriptors and four descriptor combinations showing the highest overall accuracy for the differential diagnosis of DCIS from FC.

DOR, diagnostic odds ratio; LR+/LR-, positive/negative likelihood ratio; SI: Iso- or hypointense signal intensity of the lesion compared to surrounding breast parenchyma on pre contrast $\mathrm{TI}$ or $\mathrm{T} 2$ weighted scans.
MRM in the management of DCIS remains a matter of scientific debate. Two major recent studies demonstrated that preoperative MRM adds to mammography in predicting the true extent of DCIS $(20,21)$. On the other hand, a meta-analysis pooling the data of nine older studies did not identify improvement in surgical outcomes by MRM (22).

These controversial results raise the question of how MRM of DCIS is currently interpreted in clinical practice. According to technical recommendations $(4,11)$, the centerpiece of a state of the art MRM are dynamic T1W and $\mathrm{T} 2 \mathrm{~W}$ sequences. From such images, qualitative morphologic and dynamic descriptors are extracted and based on these criteria the diagnosis is made. This was also the case for the studies referenced above $(20-22)$. If we go one step back, and question "what is the accuracy of the established criteria for the assessment of DCIS?" (11), there is clear evidence that many descriptors have a poor and not sufficient discriminatory power between malignant and benign lesions $(23,24)$.

In fact, the classification of a lesion as mass on nonmass enhancement is plausible (11); however, this approach leads to diagnostic criteria that give insufficient diagnostic accuracy $(23,24)$. As DCIS typically presents as non-mass lesions this leads to a diagnostic dilemma (11). This is why we applied a detailed checklist of 16 MRM descriptors to assess imaging patterns without the need for dichotomization of lesions into

Table 2. Pathognomonic descriptor combinations.

\begin{tabular}{|c|c|c|}
\hline Descriptor combination & DCIS & $\mathrm{FC}$ \\
\hline Continued increase + smooth margin & 0 & 100 \\
\hline $\begin{array}{l}\text { Continued increase }+ \text { irregular } \\
\text { margin }+ \text { intermediate or strong } \\
\text { wash-in }+ \text { centrifugal or centripetal } \\
\text { enhancement }\end{array}$ & 0 & 100 \\
\hline $\begin{array}{l}\text { Continued increase }+ \text { irregular margin }+ \text { weak } \\
\text { wash-in }+ \text { absence of suspicious } \\
\text { vessels* }+\mathrm{SI} \text { (T2: iso- or hypointense) }\end{array}$ & 0 & 100 \\
\hline $\begin{array}{l}\text { Wash-out or plateau }+ \text { irregular shape }+ \\
\text { SI (TI: hyperintense) }\end{array}$ & 0 & 100 \\
\hline $\begin{array}{l}\text { Wash-out }+ \text { irregular shape }+\mathrm{SI}(\mathrm{TI}: \text { iso or } \\
\text { hypointense) }+ \text { hook sign }\end{array}$ & 100 & 0 \\
\hline $\begin{array}{l}\text { Plateau }+ \text { irregular shape }+ \\
\text { SI (TI: hypointense) }\end{array}$ & 0 & 100 \\
\hline $\begin{array}{l}\text { Wash-out }+ \text { round or lobulated shape }+ \\
\text { SI (T2: hyperintense) }\end{array}$ & 0 & 100 \\
\hline
\end{tabular}

Given are all seven nodes defining pathognomonic descriptor combinations. Accordingly, each of the given descriptor combination allowed correct diagnosis of a lesion as "DCIS" or "FC" with $100 \%$ certainty. *Neither adjacent nor prominent ipsilateral vessel present. 0/I00, likelihood (\%) for the presence of DCIS or FC; SI: Signal intensity of the lesion compared to surrounding breast parenchyma as seen on $\mathrm{TI}$ (before gadolinium) or T2 scans. 


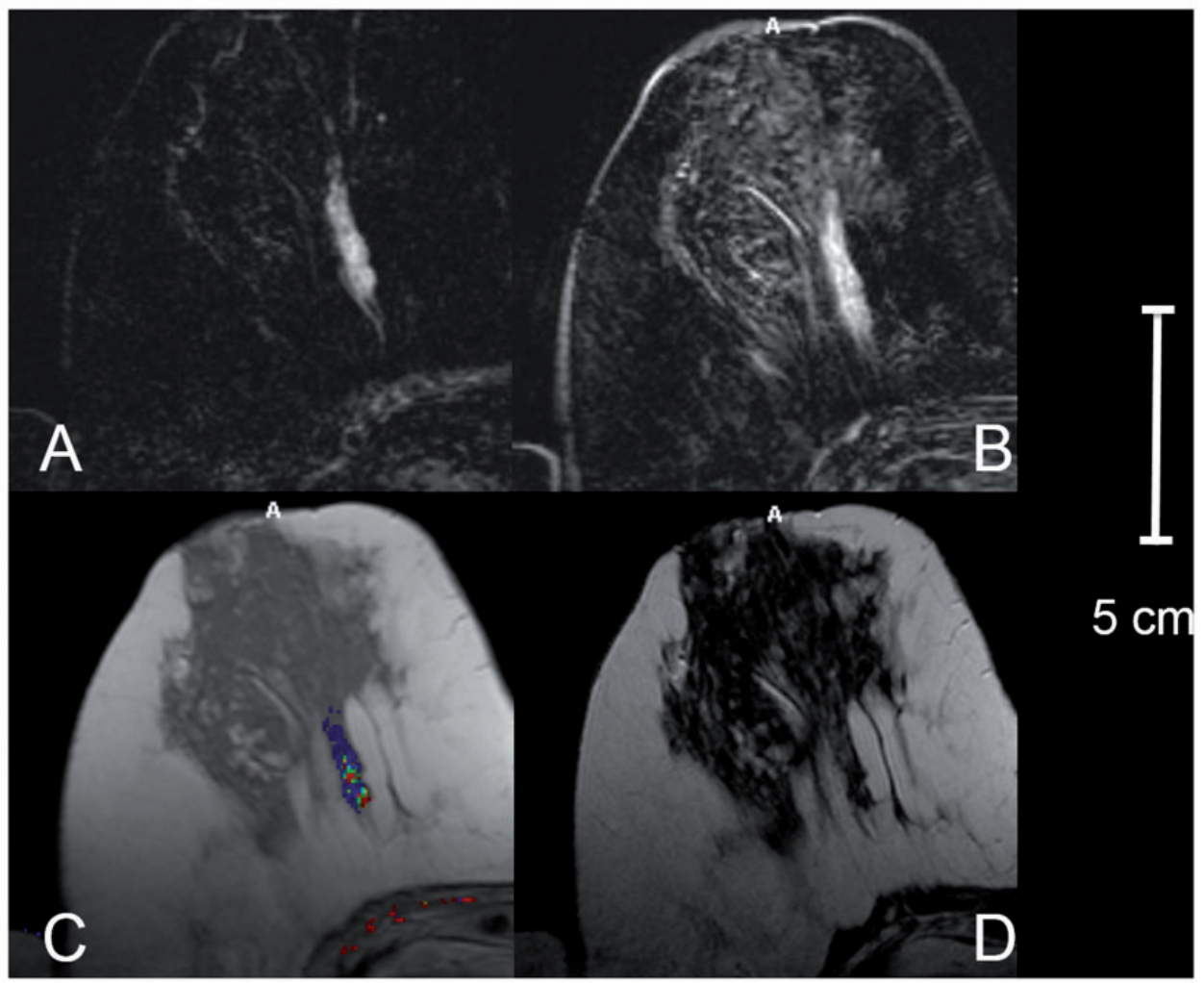

Fig. I. Classical MRM appearance of a DCIS within the dorsal parenchyma of the right breast (size $3 \mathrm{~cm}$ ). The hanging protocol from top left to bottom right: First (a) and seventh (b) subtraction of the dynamic series, TI scan pre-contrast (c), and T2 TSE scans (d). For illustrative purposes, (c) is shown with a color overlay in order to facilitate the identification of the small wash-out voxels (red). The lesion also shows strong wash-in, irregular shape (i.e. neither clearly round nor lobulated in this case), and iso- to hyperintense signal intensity on TI. These are the diagnostic criteria for node 17, providing a high specificity (96.3\%), LR + (7.5), and DOR (9.9), for the diagnosis of DCIS (Table 2). Note the additional presence of the adjacent vessel sign, further emphasizing this differential diagnosis.

mass or non-mass lesions. In a previous investigation on a more general population it has been validated, and high diagnostic accuracy with low inter-observer variability was identified (12). We could now verify the potential of this algorithm in DCIS and FC cases.

Compared to a general population including classical mass lesion, e.g. classical advanced invasive ductal cancers, we identified lower accuracy levels of MRM descriptors (13). This was to be expected, as the subgroup of DCIS and FC usually presents much more subtle features. Nevertheless 6/16 descriptors significantly distinguished FC from DCIS. What were important features?

Wash-in corresponds to tissue vascularization and was rated as "moderate" or "fast" (11) in the majority of DCIS cases (sensitivity $96.1 \%$ ). This is in accordance with both the radiological (19) as well as histological literature $(5,6)$ stating that DCIS can express pathological vascularization patterns leading to abnormal enhancement in MRM (19). Another typical finding in DCIS is periductal stromal hypervascularization promoted by tumor released cytokines $(5,6)$. This might explain the delayed peritumoral enhancement, which was depicted by the blooming sign in $41.6 \%$ of DCIS. Similarly, we could demonstrate neovascularization of DCIS in $29.9 \%$ as depicted by adjacent vessel sign. This feature gave the highest specificity $(88.6 \%)$ for the differential diagnosis of DCIS from FC, followed by the blooming sign (85.4\%). To our best knowledge, this is the first report of typical peritumoral enhancement patterns of DCIS in MRM.

However, single MRM descriptors would hardly change clinical management of lesions. This is why we applied data mining to identify diagnostic decision rules for the assessment of DCIS based on MRM descriptor combinations. This approach requires a larger sample size of DCIS cases than many previous studies and it is only surpassed by few major clinical investigations on this matter $(19,25)$. After testing on independent data by tenfold cross-validation, a total of seven nodes could be identified, enabling a definite diagnosis with $100 \%$ accuracy. From a statistical perspective, MRM could be equivalent to the gold standard (histopathologic verification), if such imagining patterns were present. 


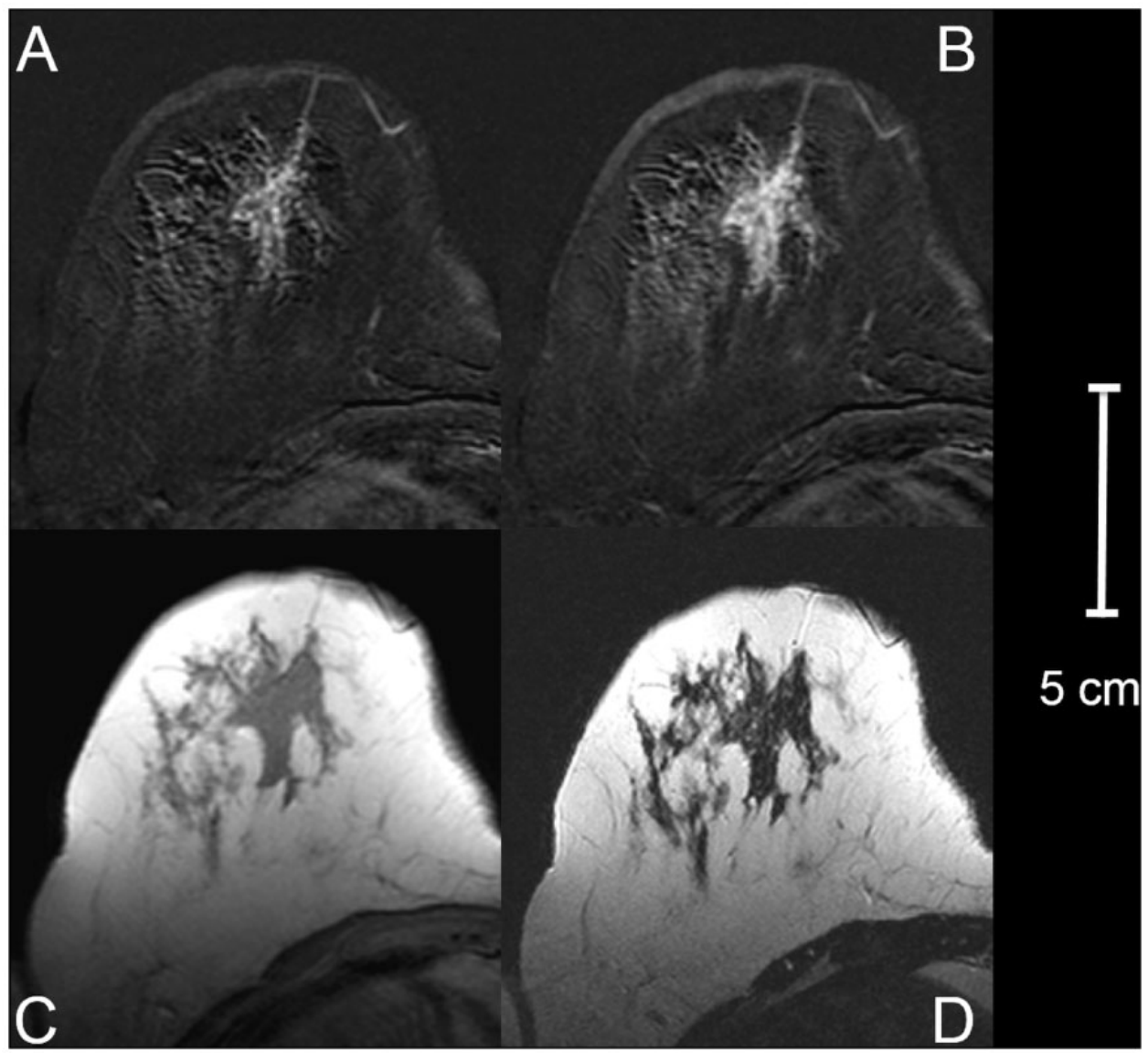

Fig. 2. Example of a ductal carcinoma in situ (DCIS) within the central parenchyma of the right breast (size up to $4 \mathrm{~cm}$ ). The hanging protocol from top left to bottom right: First (a) and seventh (b) subtraction of the dynamic series, TI scan pre-contrast (c), and T2 TSE scans (d). The lesion shows intermediate wash-in, several spots of plateau enhancement, irregular shape, isointense signal intensity $\mathrm{TI}$, and hypointense signal intensity on T2. The resulting tree node is 30 , with a specificity of $93.6 \%$ and PPV of $50 \%$ for the diagnosis of DCIS. Note the additional presence of the adjacent vessel sign and the classic blooming sign. Note also the absence of cystic components. This altogether favors the diagnosis of DCIS and histological sampling based on imaging findings is indicated.

What could be the clinical relevance of this finding? If a lesion shows a pattern pathognomonic of DCIS, there will still be a need for histopathological verification as stated by current guidelines (3). Yet, if MRM descriptors were pathognomonic for FC, biopsy could be safely avoided in our series. This finding would be more important, as the majority of the pathognomonic lesions were actually benign (FC, 94\%, 94/100) and because a significant amount of benign lesions (42.9\% of FC; 94/219) showed pathognomonic features. If our results can be verified by further clinical trials, they could decrease the biopsy rate in the workup of suspicious breast lesion in the future.

As we aimed to provide diagnostic criteria that could be applied in a standard clinical protocol, we did not evaluate diffusion-weighted imaging (DWI). This is in full agreement with current recommendations, e.g. the BI-RADS committee states that, although "there is growing evidence that (...) breast MRI could be improved by DWI (... this technique), remains in the research realm and (is) considered investigational" (11). As previous authors reported successful differentiation of DCIS by DWI (25), it is likely that this technique will play a role in the assessment of pre-invasive breast cancer in the future. This is why DWI criteria should be investigated within our diagnostic criteria and checked whether they provide additional diagnostic value. In fact, this combined reading approach of "conventional" MRM plus DWI has shown potential in the differential diagnostics of the general population (26).

Our approach was different from the standard BI-RADS descriptors. For institutions using exclusively BI-RADS descriptors, this aspect could be a possible drawback limiting direct transferability of our results. However, in this case, additional use of our interpretation algorithm could be used as an 


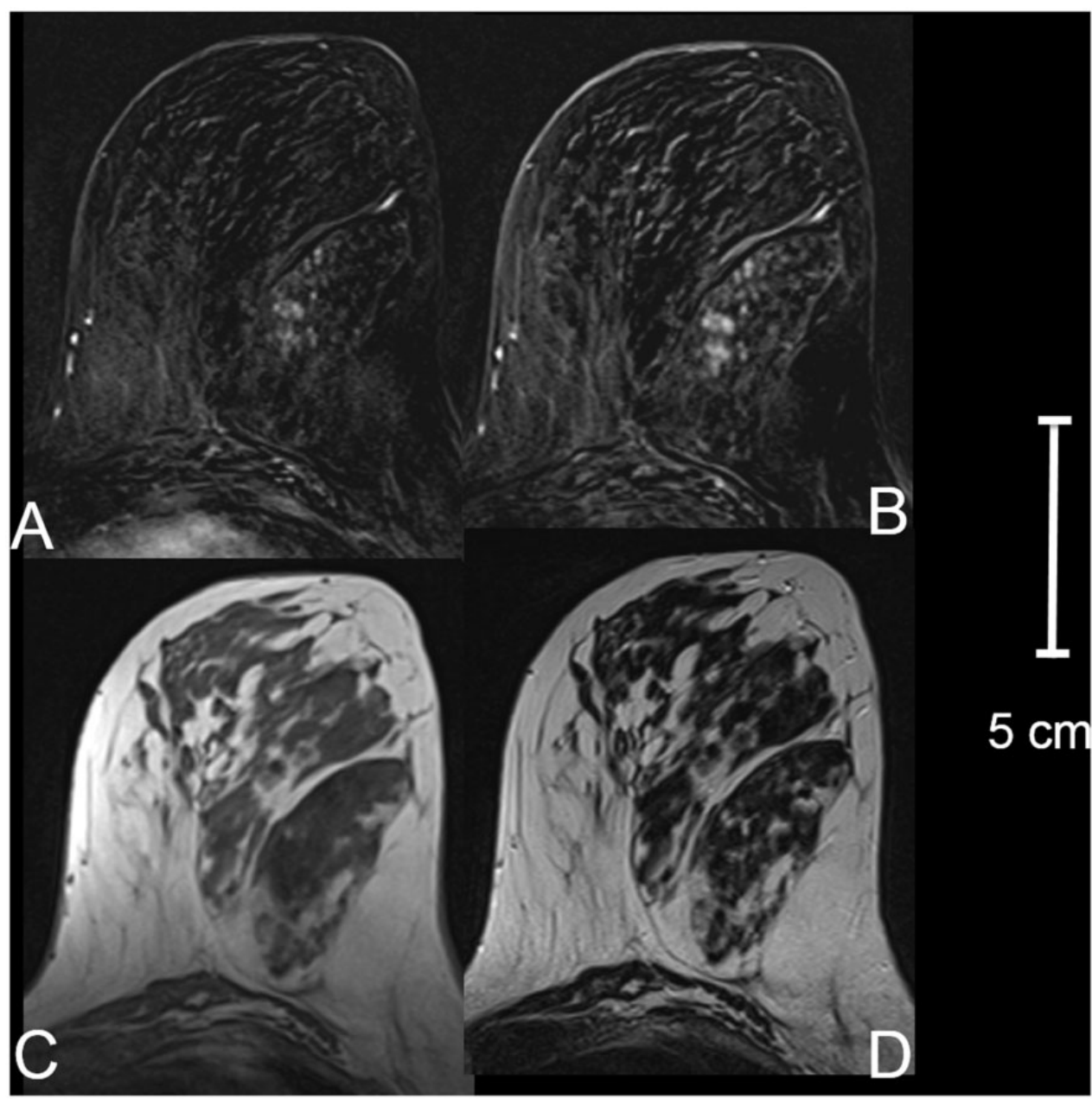

Fig. 3. True negative MRM of FC within the upper lower quadrant of the left breast (size up to $2.5 \mathrm{~cm}$ ). The hanging protocol from top left to bottom right: First (a) and seventh (b) subtraction of the dynamic series, TI scan pre-contrast (c), and T2 TSE scan (d). The lesion shows a weak wash-in plus continued signal increase and irregular margins. Additionally, the absence of suspicious vessels and the isointensity to the surrounding parenchyma on TI and T2 led to tree node 25, excluding malignancy. Note that the absence of the blooming sign further emphasizes this benign differential diagnosis.

adjunct method to generate a virtual second opinion for lesion classification.

This investigation was designed to evaluate imaging characteristics of DCIS and FC in more detail. It was not performed to subtype DCIS lesion into low-risk or high-risk groups based on imaging criteria. Currently there is active research, whether active surveillance could be a safe alternative in the management of lowrisk DCIS (27). However, neither sufficient empirical evidence exists that adjuvant therapy and/or surgery can be safely omitted in such patients, nor do we have established prognostic criteria to stratify patients into a low-risk group. Nevertheless, smaller studies have shown that MRM might play a role in this field (25). Future research should investigate whether the MRM descriptors used in the present study could stratify lesions into low-risk versus high-risk DCIS. Yet, this multivariate approach would require a significantly larger sample size, as previously shown in similar work on invasive cancers (28).

In conclusion, typical MRM descriptors for the differential diagnosis of DCIS from FC could be identified. Particularly features addressing the vasculature of the lesion's surrounding (blooming sign, adjacent vessel sign) were identified as specific parameters for this task. Furthermore, pathognomonic MRM descriptor combinations were identified enabling the diagnosis with $100 \%$ accuracy paralleling histopathology. In one-third of our patients, such pathognomonic descriptor combinations were present. As the majority of these patient groups belonged to the FC group, this approach promises to reduce biopsy rate due to false-positive imaging findings and should be further validated in clinical trials. 


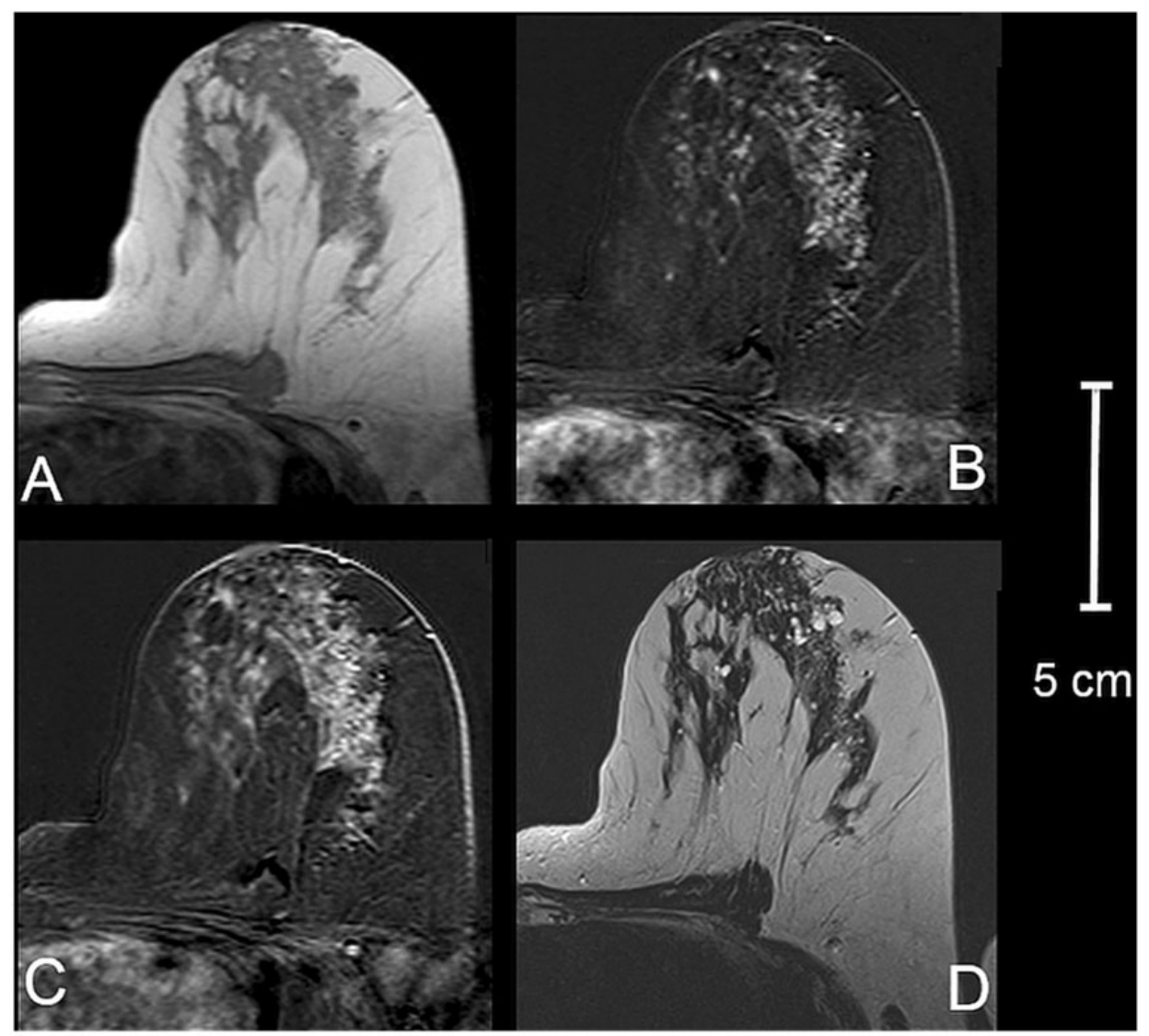

Fig. 4. Challenging case of a MRM showing fibrocystic changes (FC) within the central parenchyma of the left breast (size up to $7 \mathrm{~cm}$ ). Hanging protocol from top left to bottom right: TI scan pre-contrast (a), first (b), and seventh (c) subtraction of the dynamic series, and T2 TSE scan (d). The lesion shows an intermediate wash-in, continued increase, no wash-out, and irregular margins with an absence of centripetal or centrifugal enhancement. The T2 scans depict many cystic components and a signal intensity that is definitely hyperintense to the healthy parenchyma. These are the diagnostic criteria of node 24 , favoring with a high specificity (98.7\%) and PPV (88.9) the diagnosis of FC. Additional findings make a diagnosis of DCIS very unlikely: Despite significant lesion size, the adjacent vessel sign is absent. Multiple foci without the blooming sign during the dynamic series (a vs. b), compared to the malignant finding in Fig. 2.

\section{Acknowledgements}

The authors dedicate this work to the memory of Professor Werner Alois Kaiser, a pioneer in breast MRI. His death marked the loss of an unprejudiced, creative, and critical scientist and a remarkable clinical radiologist. The authors mourn the loss of a generous, untiring mentor and a dear and dependable friend. Prof. Kaiser carefully supervised the examination protocol adjustments used in this work in order to yield consistent image contrast and quality on different MR systems and also trained the readers of this study.

Subsets of this database have been used in clinical context previously $(14,17)$.

The authors thank Mary McAllister for her valuable help in preparing the manuscript.

\section{Declaration of Conflicting Interests}

The author(s) declared no potential conflicts of interest with respect to the research, authorship, and/or publication of this article.

\section{Funding}

The author(s) received no financial support for the research, authorship, and/or publication of this article.

\section{References}

1. Narod SA, Iqbal J, Giannakeas V, et al. Breast cancer mortality after a diagnosis of ductal carcinoma in situ. JAMA Oncol 2015;1:888-896.

2. Kuerer HM, Albarracin CT, Yang WT, et al. Ductal carcinoma in situ: state of the science and roadmap to advance the field. J Clin Oncol Off J Am Soc Clin Oncol 2009;27:279-288.

3. Sardanelli F, Boetes C, Borisch B, et al. Magnetic resonance imaging of the breast: recommendations from the EUSOMA working group. Eur J Cancer Oxf Engl 1990 2010;46:1296-1316.

4. Mann RM, Kuhl CK, Kinkel K, et al. Breast MRI: guidelines from the European Society of Breast Imaging. Eur Radiol 2008;18:1307-1318. 
5. Guidi AJ, Schnitt SJ, Fischer L, et al. Vascular permeability factor (vascular endothelial growth factor) expression and angiogenesis in patients with ductal carcinoma in situ of the breast. Cancer 1997;80:1945-1953.

6. Engels K, Fox SB, Whitehouse RM, et al. Distinct angiogenic patterns are associated with high-grade in situ ductal carcinomas of the breast. J Pathol 1997;181: 207-212.

7. Jansen SA, Paunesku T, Fan X, et al. Ductal carcinoma in situ: X-ray fluorescence microscopy and dynamic contrast-enhanced MR imaging reveals gadolinium uptake within neoplastic mammary ducts in a murine model. Radiology 2009;253:399-406.

8. Chen JH, Nalcioglu O, Su MY. Fibrocystic change of the breast presenting as a focal lesion mimicking breast cancer in MR imaging. J Magn Reson Imaging 2008;28: 1499-1505.

9. van den Bosch MAAJ, Daniel BL, Mariano MN, et al. Magnetic resonance imaging characteristics of fibrocystic change of the breast. Invest Radiol 2005;40:436-441.

10. Kreienberg R, Albert S, Folkman $M$, et al. Interdisziplinäre S3-Leitlinie für die Diagnostik, Therapie und Nachsorge des Mammakarzinoms. Langversion 3.0. Berlin: Leitlinienprogramm Onkologie, 2012. Available at: http://www.awmf.org/uploads/tx szleitlinien/032-045OL_k_S3_Brustkrebs_Mammakarzi nom_Diagnostik_Therapie_Nachsorge_2012-07.pdf (20 January 2017).

11. Morris EA, Comstock C, Lee C, et al. ACR BI-RADS ${ }^{\circledR}$ Magnetic Resonance Imaging. In: Creech WE, Priscilla FB, Wiegmann PG, et al (eds) ACR BI-RADS ${ }^{\circledR}$ Atlas Breast Imaging Report Data Syst, 5th ed. Reston, VA: American College of Radiology, 2013, pp.372-558.

12. Marino MA, Clauser P, Woitek R, et al. A simple scoring system for breast MRI interpretation: does it compensate for reader experience? Eur Radiol 2016;26:2529-2537.

13. Dietzel M, Baltzer PA, Vag T, et al. Magnetic resonance mammography in small vs. advanced breast lesions systematic comparison reveals significant impact of lesion size on diagnostic accuracy in 936 histologically verified breast lesions. RoFo 2011;183:126-135.

14. Baltzer PAT, Dietzel M, Kaiser WA. A simple and robust classification tree for differentiation between benign and malignant lesions in MR-mammography. Eur Radiol 2013;23:2051-2060.

15. Guillaume R, Taieb S, Ceugnart L, et al. BIRADS 3 MRI lesions: Was the initial score appropriate and what is the value of the blooming sign as an additional parameter to better characterize these lesions? Eur J Radiol 2016;85: $337-345$.
16. Dietzel M, Baltzer PA, Vag T, et al. Magnetic resonance mammography of invasive lobular versus ductal carcinoma: systematic comparison of 811 patients reveals high diagnostic accuracy irrespective of typing. J Comput Assist Tomogr 2010;34:587-595.

17. Dietzel M, Baltzer PAT, Vag T, et al. Application of breast MRI for prediction of lymph node metastasessystematic approach using 17 individual descriptors and a dedicated decision tree. Acta Radiol 2010;51:885-894.

18. Dietzel M, Kaiser C, Baltzer PAT. Magnetic resonance imaging of intraductal papillomas: typical findings and differential diagnosis. J Comput Assist Tomogr 2015;39: 176-184.

19. Kuhl CK, Schrading S, Bieling HB, et al. MRI for diagnosis of pure ductal carcinoma in situ: a prospective observational study. Lancet 2007;370:485-492.

20. Doyle AJ, Prakash S, Wang K, et al. DCIS of the breast: The value of preoperative MRI. J Med Imaging Radiat Oncol 2016;60:194-198.

21. Proulx F, Correa JA, Ferré R, et al. Value of pre-operative breast MRI for the size assessment of ductal carcinoma in situ. Br J Radiol 2016;89:20150543.

22. Fancellu A, Turner RM, Dixon JM, et al. Meta-analysis of the effect of preoperative breast MRI on the surgical management of ductal carcinoma in situ. Br J Surg 2015; 102:883-893.

23. Gutierrez RL, DeMartini WB, Eby PR, et al. BI-RADS lesion characteristics predict likelihood of malignancy in breast MRI for masses but not for nonmasslike enhancement. Am J Roentgenol 2009;193:994-1000.

24. Baltzer PAT, Benndorf $M$, Dietzel $M$, et al. Falsepositive findings at contrast-enhanced breast MRI: a BI-RADS descriptor study. Am J Roentgenol 2010;194: 1658-1663.

25. Rahbar H, Partridge SC, Eby PR, et al. Characterization of ductal carcinoma in situ on diffusion weighted breast MRI. Eur Radiol 2011;21:2011-2019.

26. Baltzer A, Dietzel M, Kaiser CG, et al. Combined reading of Contrast Enhanced and Diffusion Weighted Magnetic Resonance Imaging by using a simple sum score. Eur Radiol 2015;26:884-891.

27. Elshof LE, Tryfonidis K, Slaets L, et al. Feasibility of a prospective, randomised, open-label, international multicentre, phase III, non-inferiority trial to assess the safety of active surveillance for low risk ductal carcinoma in situ-The LORD study. Eur J Cancer Oxf Engl 1990 2015;51:1497-1510.

28. Dietzel M, Baltzer PA, Vag T, et al. Potential of MR mammography to predict tumor grading of invasive breast cancer. RoFo 2011;183:826-833. 